\title{
Early Management of Scars Using a 532-nm Nd:YAG Laser
}

Jun-Young Jang ${ }^{1}$, Jin Ho Han ${ }^{2}$, Kun Chul Yoon', Hyun Woo Shin'2, Yong Seong Kim ${ }^{2}$, June-Kyu Kim ${ }^{2}$

${ }^{1}$ Seodo Public Health Care Center, Incheon; ${ }^{2}$ Department of Plastic and Reconstructive Surgery, Kangbuk Samsung Medical Center, Sungkyunkwan University School of Medicine, Seoul, Korea

No potential conflict of interest relevant to this article was reported.
Background Laser therapy is generally postponed until scar maturation. We speculated that the use of laser therapy at the beginning of the wound healing process might minimize discomfort and problems at an early stage.

Methods From January 2013 to May 2014, we conducted a study of 50 patients who underwent primary closure or scar revision procedures due to facial scarring. Within 1 to 2 weeks after the removal of all stitches, we performed laser treatments with a 532$\mathrm{nm}$ neodymium-doped yttrium aluminium garnet (Nd:YAG) laser. To assess the improvement of the scars, we evaluated the scars using the Vancouver Scar Scale (VSS) and conducted a photo analysis using a computer graphics program.

Results The mean score on the VSS decreased from 5.99 to 1.49 after the laser treatment. The score for each evaluation item decreased, meaning that the scars improved. The differences showed statistical significance. In the photo analysis that was conducted using Adobe Photoshop, the mean brightness of the pixels located within the scar territory increased from $61.54 \%$ to $69.42 \%$ after laser treatment, and the mean chroma decreased from $50.65 \%$ to $43.12 \%$. These changes were statistically significant. Conclusions We used a 532-nm Nd:YAG laser at an early stage of scar formation and confirmed improvements in the redness, hyperpigmentation, and hypertrophy of the scars shortly after treatment.

Keywords Cicatrix, Laser therapy, Lasers

\section{INTRODUCTION}

Facial scars cause individuals to experience psychological stress and social withdrawal, reducing their quality of life [1-7]. In order to minimize facial scars, a wide range of treatment methods have been developed and utilized. These techniques include surgical scar revision, steroid injections, silicone gel and sheet application, cryosurgery, anti-cancer drug injections (e.g., 5-fluorouracil or bleo-

Received: Apr 19, 2017 Revised: May 25, 2017 Accepted: May 25, 2017 Correspondence: June-Kyu Kim Department of Plastic and Reconstructive Surgery, Kangbuk Samsung Medical Center, Sungkyunkwan University School of Medicine, 29 Saemunan-ro, Jongno-gu, Seoul 03181, Korea. E-mail: kokoro72@naver.com

Copyright () 2017 The Korean Society for Aesthetic Plastic Surgery.

This is an Open Access article distributed under the terms of the Creative Commons Attribution Non-Commercial License (http://creativecommons.org/licenses/by-nc/4.0/) which permits unrestricted non-commercial use, distribution, and reproduction in any medium, provided the original work is properly cited. www.e-aaps.org mycin), and laser irradiation [8-13].

Among these techniques, laser therapy is considered the least invasive. In 1993, a pulsed dye laser was first used for scar treatments, and many treatments and procedures have since been developed with different light sources, wavelengths, and durations [14-21].

Laser therapy is generally postponed until scar maturation. Therefore, it is quite common for patients to suffer due to the redness, hyperpigmentation, and hypertrophy of the scar. We thus speculated that the use of laser therapy at the beginning of the wound healing process might minimize these problems at an early stage.

A 532-nm frequency-doubled Q-switched neodymium-doped yttrium aluminium garnet (Nd:YAG) laser is commonly used to treat superficial pigmented lesions (e.g., postinflammatory hyperpigmentation, melasma, lentigines, café au lait macules, Becker nevus, ephelides, and tattoos) [22]. Some studies have shown that the 532-nm wavelength is easily absorbed by hemoglobin and melanin materials, which can be beneficial for the improvement of redness, 
hyperpigmentation, and hypertrophy in scars [23]. Therefore, we selected the 532-nm frequency-doubled Q-switched Nd:YAG laser as our early treatment tool, hypothesizing that this laser might be effective for minimizing the redness, hyperpigmentation, and hypertrophy that occur early in scar formation.

\section{METHODS}

Within 1 to 2 weeks after the removal of all stitches, we performed laser treatment in patients who had undergone primary closure or scar revision procedures. To assess the improvement of the scars, we evaluated the scars using the Vancouver Scar Scale (VSS) and conducted a photo analysis using a computer graphics program (Adobe Photoshop CS6; Adobe Inc., San Jose, CA, USA) 2 weeks after the last round of laser irradiation.

\section{Patient selection}

From January 2013 to May 2014, we conducted a study of 50 patients who underwent primary closure or scar revision procedures due to facial scarring. Prior to the study, researchers obtained informed consent from each patient and all ethical guidelines were followed.

The patients consisted of 40 men and 10 women, with an average age of 38 years (range, 14-62 years). Forty-five patients underwent a primary closure procedure due to facial lacerations caused by a traumatic incident. Five patients required a scar revision because of facial scarring. All patients received laser treatments within 2 weeks of suture removal (Table 1).

\section{Taking pictures in a standard setting}

Pictures were taken before laser therapy and additionally at every follow-up outpatient visit until the final round of laser therapy. All pictures were taken under conditions that were as similar as possible to compare the scars before and after laser therapy. Before taking pictures, certain procedures were avoided, such as irritating the skin by applying a lidocaine ointment or cleansing the patients' skin. In addition, researchers required patients to rest for at least $10 \mathrm{~min}$ utes to prevent the occurrence of redness or paleness caused by skin

Table 1. Demographics of patients

\begin{tabular}{lc}
\hline Variable & Value $(\mathrm{n}=50)$ \\
\hline Age lyear) & $38.07 \pm 14.46(14-62)$ \\
Sex & $40(80 \%)$ \\
Male & $10(20 \%)$ \\
Female & \\
Cause of scar & $45(90 \%)$ \\
Laceration & $5(10 \%)$ \\
Scar revision & \\
\hline
\end{tabular}

irritation. A photographer took the patients' pictures using a Canon EOS 600 D camera (Canon Inc., Tokyo, Japan) and care was taken to maintain identical conditions; the same room, lighting, location, posture, and camera settings were used.

\section{Laser treatment}

The laser treatment was performed with a 532-nm Nd:YAG laser (Spectra VRM III; Lutronic, Goyang, Korea) within 1 to 2 weeks after all stitches were removed. The energy density of the laser was less than $1 \mathrm{~J} / \mathrm{cm}^{2}\left(0.6-0.8 \mathrm{~J} / \mathrm{cm}^{2}\right)$, the spot size was $2.6 \mathrm{~mm}$, and the pulse duration was $80 \mathrm{~ns}$.

After the laser treatment, patients were instructed to apply a prescribed antibiotic ointment daily for up to 3 days. They were also advised to avoid sunlight and physical irritation during the treatment period. Patients received the laser treatment at fortnightly intervals. After a comparison with previous pictures, if the treatment appeared to be effective for the patient, we performed additional

Table 2. Laser treatment data

\begin{tabular}{lc}
\hline Variable & Value $(\mathrm{n}=50)$ \\
\hline $\begin{array}{l}\text { Interval from stitch removal to initial laser } \\
\text { treatment (day) }\end{array}$ & $9.1 \pm 2.9(5-13)$ \\
Follow-up time (day) & $42.2 \pm 32.2(14-122)$ \\
Rounds of laser treatment & $4 \pm 1.3(2-6)$ \\
Complications & None \\
\hline
\end{tabular}

Table 3. Vancouver Scar Scale

\begin{tabular}{|c|c|}
\hline Items & \\
\hline \multirow[t]{3}{*}{ Pigmentation } & 0: normal \\
\hline & 1: hypopigmentation \\
\hline & 2: hyperpigmentation \\
\hline \multirow[t]{6}{*}{ Vascularity } & 0: normal \\
\hline & 1: pink \\
\hline & 2: pink to red \\
\hline & 3: red \\
\hline & 4: red to purple \\
\hline & 5: purple \\
\hline \multirow[t]{6}{*}{ Pliability } & $0:$ normal \\
\hline & 1: supple \\
\hline & 2: yielding \\
\hline & 3: firm \\
\hline & 4: banding \\
\hline & 5: contracture \\
\hline \multirow[t]{4}{*}{ Height } & 0: normal \\
\hline & $1:<2 \mathrm{~mm}$ \\
\hline & $2: 2-5 \mathrm{~mm}$ \\
\hline & $3:>5 \mathrm{~mm}$ \\
\hline
\end{tabular}


treatment. However, if the efficacy of the laser therapy was not distinguishable or if the pigmentation and vascularity had mostly improved, the therapy was stopped. Patients received an average of 4 rounds of treatment (range, 2-6). The laser therapy treatments were completed after an average of 6 weeks (42 days) (Table 2).

\section{Scar evaluation}

To evaluate the improvement of the patients' scars, we used the following methods: 1) macroscopic scar evaluation using the VSS and 2) photo analysis using Adobe Photoshop.

\section{Physical examination and survey}

The VSS assesses scars according to 4 items: pigmentation, vascularity, pliability, and height. A score of 0 indicates normal skin. Higher scores correspond to more severe scars (Table 3). Three plastic surgeons assessed the patients' scars using the VSS before and after treatment, and we compared the scores.

\section{Photoshop}

We analyzed pictures taken before and after treatment using Adobe
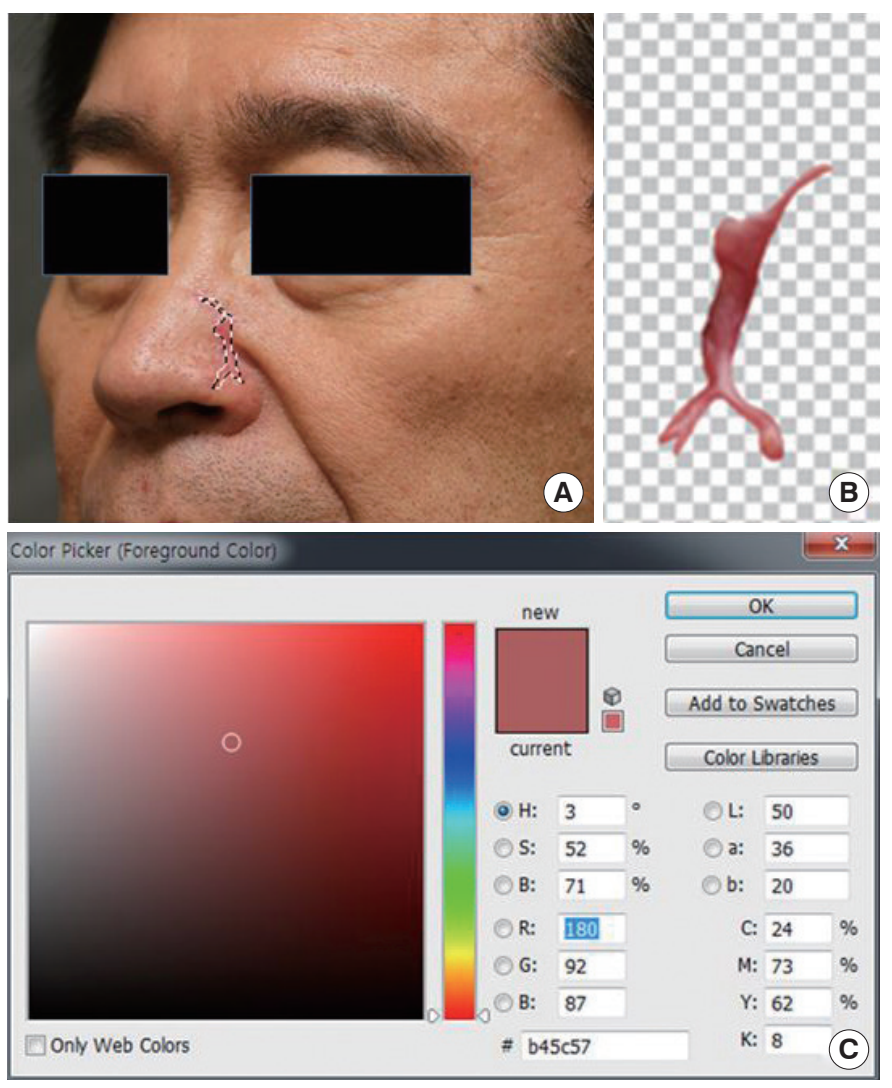

Fig. 1. Photo analysis using Adobe Photoshop (Adobe Systems Inc., San Jose, CA, USA). (A) According to the scar margin within a photo, the scar territory was designated. (B) The region beyond the scar territory was removed. (C) The mean values of the pixels were calculated within the scar territory.
Photoshop. A pixel is the smallest unit comprising a picture and is composed of brightness, chroma, and color. Brightness is the degree of the color's luminance, chroma is the strength of an object's color, and color represents the unique attributes of the color. We designated a territory corresponding to the scar margin within each photo and computed the average brightness and chroma of all pixels within that territory (Fig. 1). In addition, brightness and chroma values were measured on normal skin at least $2 \mathrm{~cm}$ away from the scar territory and used as controls. We then observed changes in brightness and chroma after the laser treatment.

\section{Statistical analysis}

Statistical analysis was conducted using SPSS version 24 (IBM Corp., Armonk, NY, USA), and the t-test was used to assess statistical significance.

\section{RESULTS}

The mean score of the VSS decreased from 5.99 to 1.49 after the laser treatment. The score for each evaluation item (pigmentation, vascularity, pliability, and height) decreased, meaning that the scars improved (Table 4). The differences showed statistical significance $(\mathrm{P}<0.05)$ (Fig. 2).

In the photo analysis using Adobe Photoshop, the mean brightness of the pixels located within the scar territory increased from $61.54 \%$ to $69.42 \%$ after the laser treatment $(\mathrm{P}<0.05)$, and the mean chroma decreased from $50.65 \%$ to $43.12 \%$ (Table 5 ). These changes were statistically significant (Fig. 3). These changes indicate that the patients had brighter skin after the laser treatment than before receiving the treatment. Furthermore, as the chroma of the scars decreased, the redness disappeared and the hyperpigmentation improved as well. Meanwhile, the chroma values and the brightness values measured in the control group did not significantly change after treatment.

Table 4. Results of the Vancouver Scar Scale

\begin{tabular}{lccc}
\hline Variable & $\begin{array}{c}\text { Before laser } \\
\text { treatment }\end{array}$ & $\begin{array}{c}\text { After laser } \\
\text { treatment }\end{array}$ & P-value \\
\hline Pigmentation & $1.88 \pm 0.38$ & $0.97 \pm 0.21$ & $<0.001$ \\
Vascularity & $2.08 \pm 0.58$ & $0.33 \pm 0.31$ & $<0.001$ \\
Pliability & $1.17 \pm 0.64$ & $0.05 \pm 0.15$ & $<0.001$ \\
Height & $0.86 \pm 0.48$ & $0.13 \pm 0.30$ & $<0.001$ \\
\hline
\end{tabular}

Table 5. Photoshop analysis

\begin{tabular}{lccc}
\hline Variable & $\begin{array}{c}\text { Before laser } \\
\text { treatment }\end{array}$ & $\begin{array}{c}\text { After laser } \\
\text { treatment }\end{array}$ & P-value \\
\hline Chroma & $50.65 \pm 12.03(28-72)$ & $43.11 \pm 11.12(27-69)$ & $<0.001$ \\
Brightness & $61.54 \pm 10.92(44-76)$ & $69.42 \pm 10.74(46-85)$ & $<0.001$ \\
\hline
\end{tabular}



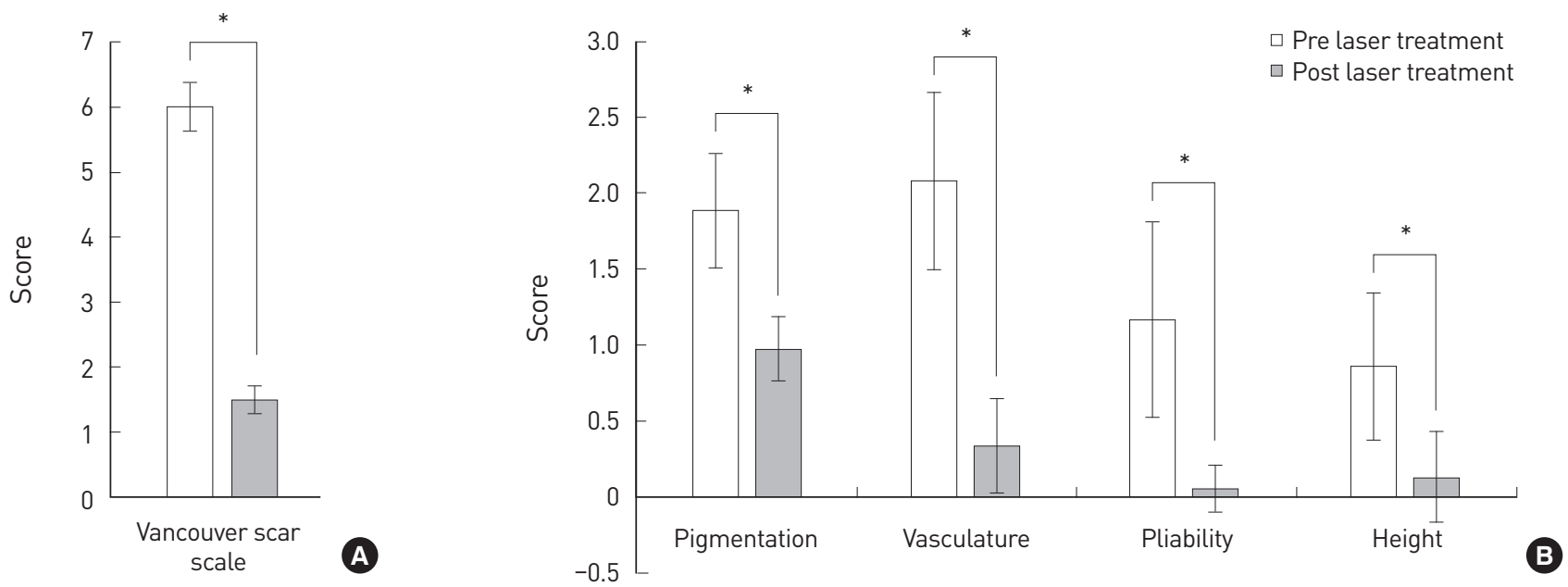

Fig. 2. Scar evaluation using the Vancouver Scar Scale (VSS). (A) Total VSS score before and after the final laser treatment ( $n=50$ ); $P<0.01$. (B) Individual VSS scores before and after the final laser treatment ( $n=50) ; P<0.01$.

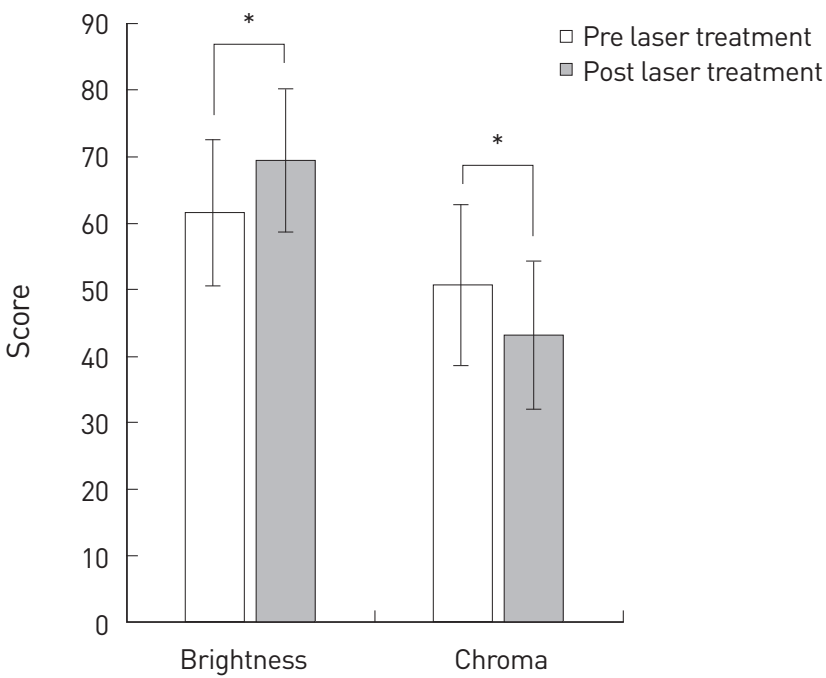

Fig. 3. Scar evaluation using Adobe Photoshop (Adobe Systems Inc., San Jose, CA, USA). After laser treatment, the average brightness of the pixels within the scar territory increased and the average chroma of the pixels decreased ( $n=50)$; $P<0.01$.

\section{DISCUSSION}

Although many treatment modalities have been developed and used to improve scars, most management options have generally been initiated after scar maturation. There have been few attempts to manage scars at an early stage. Thus, patients with scars that exhibit severe redness and hyperpigmentation must endure discomfort and wait for up to 6 months until the scars mature. We performed laser irradiation at the beginning of the wound healing process to improve the redness, hyperpigmentation, and hypertrophy of scars at an early stage.
Wound healing occurs in the following phases: inflammation, proliferation, and remodeling. A scar is the final product of the remodeling phase and matures over the course of a few months to several years [5]. In immature scar tissue, redness, hyperpigmentation, and hypertrophy tend to be more apparent during the initial 2 to 3 months. However, after going through a static phase, scars mature as they change to a lighter color and begin to flatten [24]. However, some scars retain redness, hyperpigmentation, and hypertrophy over several months or years.

During the proliferation phase, beginning 5 days after the initial wound, fibroblasts and macrophages begin to enter and deposit around the wound site. Afterward, a dense capillary network induced by angiogenesis is formed. The fibroblasts of this capillary network supply nutrients for the collagen synthesis process to support the wound healing process.

Dense capillary networks cause redness at an early stage of the wound healing process. However, over time, scars begin to regress naturally and the redness of the scars gradually disappears. However, during this maturation phase, if the dense capillary networks fail to regress, redness will remain noticeable. Scar hypertrophy also occurs as a result of continuous nourishment from the remaining capillary networks and collagen biosynthesis by fibroblasts. In addition, the excessive proliferation of melanin leads to hyperpigmentation [23].

We hypothesized that by controlling these wound healing processes, we would be able to improve the redness, hyperpigmentation, and hypertrophy of scars. A 532-nm Nd:YAG laser was used because the 532-nm wavelength is easily absorbed by hemoglobin and melanin, so that the laser can selectively destroy both of these substances. Bowes et al. [23] published a research paper stating that they achieved improvements in pigmented hypertrophic scars after maturation with a 532-nm Nd:YAG laser. 

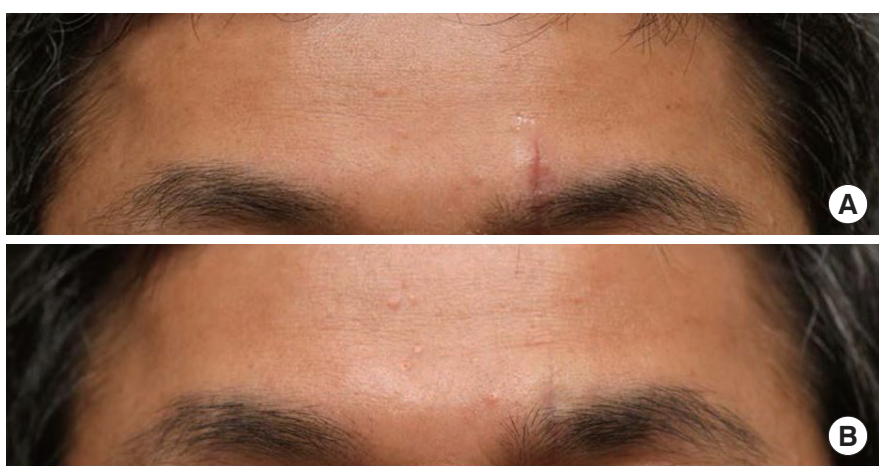

Fig. 4. Case. A 52-year-old male patient received primary closure and stitch removal 5 days subsequently due to a 2-cm-deep muscle laceration in the left eyebrow from a traumatic incident. One week later, he was followed up at an outpatient clinic. (A) He received 532$\mathrm{nm}$ neodymium-doped yttrium aluminium garnet ( $\mathrm{Nd}: \mathrm{YAG}$ ) laser treatment 3 times at intervals of 1 to 2 weeks to reduce the redness, hyperpigmentation, and mild hypertrophy in the scar. (B) We confirmed that after 35 days, the redness, hyperpigmentation, and hypertrophy in the scar had improved.

The 532-nm Nd:YAG laser only penetrates into the superficial dermis, where the vessels of hypertrophic or erythematous scars are located. Therefore, as 532-nm laser beams selectively destroy oxyhemoglobin and cause microvascular injuries, proliferating capillary networks are reduced, which improves the redness of scars. Furthermore, microvessel injuries prevent fibroblasts from receiving nourishment and synthesizing excessive collagen, reducing the production of hypertrophic scars [8,9]. Moreover, absorption of the energy in melanocytes or melanosomes hinders the excessive proliferation of melanin and prevents hyperpigmentation [23].

Theoretically and practically, redness, hyperpigmentation, and hypertrophy in many patients may worsen after 2 or 3 months. Using a 532-nm Nd:YAG laser, the early wound healing process was influenced and satisfactory results were achieved within 6 weeks (42 days) on average after treatment (Fig. 4). Thus, it is unnecessary to wait for the spontaneous improvement of scars.

When we evaluated scars using the VSS after laser treatment, statistically significant decreases in all 4 parameters (pigmentation, vascularity, pliability, and height) were observed. These results suggest that the 532-nm Nd:YAG laser is generally effective for improving hyperpigmentation, redness, and hypertrophy in scars.

Although the VSS is one of the most widely used tools for scar evaluation, it has limitations, as it relies only on an evaluator's naked eye, meaning that it can be somewhat subjective. Therefore, we conducted a photo analysis using Adobe Photoshop in order to make objective observations of the changes that occurred as scars improved.

Through the photo analysis, we confirmed that the average brightness of pixels increased within the scar territory, while the chroma decreased. These results were statistically significant, meaning that compared to normal skin, vascularity was higher and more pigmentation occurred in hypertrophic pigmented scars. Once a scar improved, the redness and hyperpigmentation also improved, as indicated by an increase in brightness and a decrease in chroma.

Although the photo analysis using Adobe Photoshop has the disadvantage of not being systematized and standardized, it has the potential to become a valuable scar evaluation tool in the future. This is because quantification of the improvement of scars is feasible by comparing brightness and chroma values using a computer graphics program.

One of the biggest limitations of this study is the lack of a control group. Thus, the efficacy of laser treatments could not be comprehensively established, as we did not compare scar improvement over time between a group that underwent laser treatment and another group that did not. However, this study demonstrated that laser treatment improved the redness, hyperpigmentation, and hypertrophy of scars at an early stage.

\section{CONCLUSION}

We used a 532-nm Nd:YAG laser at an early stage of scar formation and confirmed improvements in the redness, hyperpigmentation, and hypertrophy of scars within a short period. Thus, we achieved the following satisfactory results: scars were improved after an average of 4 laser treatments, and the achievement of quick therapeutic effects reduced the length of time that patients suffered while waiting for scars to mature. Additionally, through a photo analysis conducted using Adobe Photoshop, we objectively confirmed that the scars improved. We suggest that this method may be able to serve as a new tool to compare scars objectively if it is standardized in further studies.

\section{PATIENT CONSENT}

Patients provided written consent for the use of their images.

\section{REFERENCES}

1. Levine E, Degutis L, Pruzinsky T, et al. Quality of life and facial trauma: psychological and body image effects. Ann Plast Surg 2005;54:502-10.

2. Robert R, Meyer W, Bishop S, et al. Disfiguring burn scars and adolescent self-esteem. Burns 1999;25:581-5.

3. Tebble NJ, Adams R, Thomas DW, et al. Anxiety and self-consciousness in patients with facial lacerations one week and six months later. Br J Oral Maxillofac Surg 2006;44:520-5.

4. Young VL, Hutchison J. Insights into patient and clinician concerns about scar appearance: semiquantitative structured surveys. Plast Reconstr Surg 2009;124:256-65.

5. Bisson JI, Shepherd JP, Dhutia M. Psychological sequelae of facial trauma. J Trauma 1997;43:496-500. 
6. Bryant RA, Harvey AG. Initial posttraumatic stress responses following motor vehicle accidents. J Trauma Stress 1996;9:223-34.

7. Smith H. Challenging disfigurement. Community Pract 2000;73:637.

8. Tziotzios C, Profyris C, Sterling J. Cutaneous scarring: Pathophysiology, molecular mechanisms, and scar reduction therapeutics Part II. Strategies to reduce scar formation after dermatologic procedures. J Am Acad Dermatol 2012;66:13-24.

9. Eming SA, Krieg T, Davidson JM. Inflammation in wound repair: molecular and cellular mechanisms. J Invest Dermatol 2007;127:514-25.

10. Gold MH. Dermabrasion in dermatology. Am J Clin Dermatol 2003; 4:467-71.

11. Musgrave MA, Umraw N, Fish JS, et al. The effect of silicone gel sheets on perfusion of hypertrophic burn scars. J Burn Care Rehabil 2002;23: 208-14.

12. Bouzari N, Davis SC, Nouri K. Laser treatment of keloids and hypertrophic scars. Int J Dermatol 2007;46:80-8.

13. Elsaie ML, Choudhary S, McLeod M, et al. Scars. Curr Probl Dermatol 2011;42:131-9.

14. Alster TS, Kurban AK, Grove GL, et al. Alteration of argon laser-induced scars by the pulsed dye laser. Lasers Surg Med 1993;13:368-73.

15. Nouri K, Jimenez GP, Harrison-Balestra C, et al. 585-nm pulsed dye laser in the treatment of surgical scars starting on the suture removal day. Dermatol Surg 2003;29:65-73.

16. Nouri K, Rivas MP, Stevens M, et al. Comparison of the effectiveness of the pulsed dye laser $585 \mathrm{~nm}$ versus $595 \mathrm{~nm}$ in the treatment of new surgical scars. Lasers Med Sci 2009;24:801-10.

17. Nouri K, Elsaie ML, Vejjabhinanta V, et al. Comparison of the effects of short- and long-pulse durations when using a 585-nm pulsed dye laser in the treatment of new surgical scars. Lasers Med Sci 2010;25: $121-6$.

18. Conologue TD, Norwood C. Treatment of surgical scars with the cryogen-cooled $595 \mathrm{~nm}$ pulsed dye laser starting on the day of suture removal. Dermatol Surg 2006;32:13-20.

19. Jung JY, Choi YS, Yoon MY, et al. Comparison of a pulsed dye laser and a combined 585/1,064-nm laser in the treatment of acne vulgaris. Dermatol Surg 2009;35:1181-7.

20. Berlin AL, Hussain M, Goldberg DJ. Cutaneous photoaging treated with a combined 595/1064 nm laser. J Cosmet Laser Ther 2007;9:214-7.

21. Trelles MA, Weiss R, Moreno-Moragas J, et al. Treatment of leg veins with combined pulsed dye and Nd:YAG lasers: 60 patients assessed at 6 months. Lasers Surg Med 2010;42:609-14.

22. Lask GP, Glassberg E. Neodymium:yttrium-aluminum-garnet laser for the treatment of cutaneous lesions. Clin Dermatol 1995;13:81-6.

23. Bowes LE, Nouri K, Berman B, et al. Treatment of pigmented hypertrophic scars with the $585 \mathrm{~nm}$ pulsed dye laser and the $532 \mathrm{~nm}$ frequency-doubled Nd:YAG laser in the Q-switched and variable pulse modes: a comparative study. Dermatol Surg 2002;28:714-9.

24. Mustoe TA, Cooter RD, Gold MH, et al. International clinical recommendations on scar management. Plast Reconstr Surg 2002;110:56071. 